\title{
Enhancing dialogues between rehabilitation patients and therapists using visualisation software
}

\author{
David Loudon \\ The Glasgow School of Art \\ Glasgow, UK \\ d.loudon@gsa.ac.uk
}

\author{
Alastair S. Macdonald \\ The Glasgow School of Art \\ Glasgow, UK \\ a.macdonald@gsa.ac.uk
}

\begin{abstract}
This paper discusses different ways of enhancing patient-therapist dialogues using a new visualisation tool for rehabilitation, which provides patients with a visual and interactive interface to observe and understand their own movements. The opportunities for using the tool in both clinical and home environments are discussed.
\end{abstract}

\section{visualisation; rehabilitation; patient understanding}

\section{INTRODUCTION}

The potential to transfer some aspects of the rehabilitation process into the home using new technologies is becoming feasible due to the increasing availability of lower cost alternatives for expensive, lab-based rehabilitation equipment. If this potential can be realised, it could lead to cost savings for the delivery of rehabilitation services, and benefit the patient by enabling them to perform their rehabilitation at their convenience, in the familiarity of their own environment. However, by changing the way rehabilitation services are delivered it will be crucial to recognise the importance of sufficient levels of interaction between the patient and the therapist [1].

This paper discusses the potential for new technologies to enhance the dialogue between patients and therapists, rather than replace it. A new visualisation tool for rehabilitation is described, which provides patients with a visual and interactive interface to observe and understand their own movements. The opportunities to enhance dialogue will be discussed in relation to the use of the tool in a clinical setting, before contrasting the opportunities and limitations of its use in the home environment.

\section{BACKGROUND}

\section{A. Visualisation of biomechanics for rehabilitation}

For many physical rehabilitation issues a biomechanical understanding of the problem and its solution is essential [2]. However, despite more than three decades of developments in the field, the potential of biomechanics to fully influence healthcare has remained under-exploited due to the problematic nature of communicating complex biomechanical data to other disciplines and to lay people.

A previous study by the authors [3] investigated the ability of professionals from different disciplinary backgrounds and lay persons to understand example visualisations of complex biomechanical information. The research found that through the use of visualisation techniques data which would usually have been inaccessible could be understood by both lay and professional audiences. Further, the visualisations were shown to enable new cross-disciplinary dialogues about the data between the professionals and lay members.

The envisage project, funded by the Lifelong Health and Wellbeing (LLHW2) initiative, builds on this previous research, and will investigate the potential of visualisations of biomechanical data to improve rehabilitation services and the treatments they deliver to patients. The project is a multidisciplinary collaboration between the University of Strathclyde, The Glasgow School of Art and Glasgow Caledonian University.

Six discrete work packages have been selected to investigate the application of the visualisation tool to a range of rehabilitation processes and complexity of condition.

- Exercise advice to the healthy older adult

- Falls prevention advice and visual feedback to those at risk of falling.

- Functional exercises for the rehabilitation of total knee replacement patients

- Lower limb stroke rehabilitation for acute stroke patients.

- Upper limb stroke rehabilitation for acute stroke patients.

- Diagnosis and fitting of an ankle foot orthosis in late stage stroke.

Each work package will evaluate the effectiveness of the visualisation tool intervention on patient outcomes in a Phase II randomised clinical trial (exploratory) as defined by the MRC and will follow MRC guidelines for the evaluation of complex interventions [4].

In accordance with the guidelines, an essential and integrated component to the project will be to explore, using qualitative methods, the effects of the use of the tool on the experiences of both the patients and therapists and the interactions between the different participants [5]. 


\section{B. Flexible design of the visualisation tool}

Building on the effectiveness of the prototype visualisation tool from previous research [6], a new flexible, fully customisable visualisation software tool is being developed, which will enable the exploration of different visual techniques to provide biomechanical information relevant to the rehabilitation concerns of the patients and therapists. The tool is flexible in two ways.

Firstly, the tool is flexible in the selection of the data input technology used. The tool will use motion capture data of the individual patients to generate visualisations of data relevant to their rehabilitation. The software will be used with a range of motion capture equipment: from expensive laboratory setups to small custom sensors suitable for the home. The tool has therefore been designed to work independently of which motion capture technology is used. This also leaves open the option of integrating any new advances in motion capture technology or lower cost equipment which may emerge over the course of the project. The use of motion capture data collected in both real-time and 'off-line' will be supported by the tool.

Secondly, the tool is flexible in terms of the interface used and the visualisation options presented to the patient. Six different variations of the tool will be created, tailored to the different requirements of the patients and therapists participating in the work packages and the biomechanical information to be communicated.

In addition, the flexibility of the tool will enable different visualisation options to be iteratively developed in response to feedback from representative stakeholders for each of the work packages [12]. Four focus groups will be held with: stroke survivors; stroke professionals; older adults; and professionals working with older adult exercise. The aim in these sessions will be to explore how the stakeholders understand early prototypes of the visualisations, in order to inform the design of the RCT tools [13]. Following the focus groups, pre-RCT pilots will be held with a smaller group of representative users in order to explore the interaction with the visualisation tool as intended in the RCT [14].

\section{EXAMPLE SCENARIOS OF PATIENT - THERAPIST DIALOGUES USING THE VISUALISATION TOOL}

Different scenarios of interaction between therapists and patients will be explored across the work packages in the project. In this section, three example scenarios are chosen to illustrate a subset of the interactions which will be investigated:

- the use of the tool to enable communication and discussion of rehabilitation tasks and goals

- the use of the tool to enable communication and discussion of the progress of the patient towards their rehabilitation goals

- the use of the tool independently by the patient in the home, in order to maintain patient motivation and adherence to their rehabilitation tasks

\section{A. Scenario 1: Communication and discussion of patient's rehabilitation tasks and goals}

In this scenario an acute post-stroke patient has been asked to do an upper arm rehabilitation reaching task. The patient has just completed the task and both patient and therapist are sitting down in front of the screen to play back the movement. Figure 1 shows a screen shot from an early prototype of how this may appear to the patient.

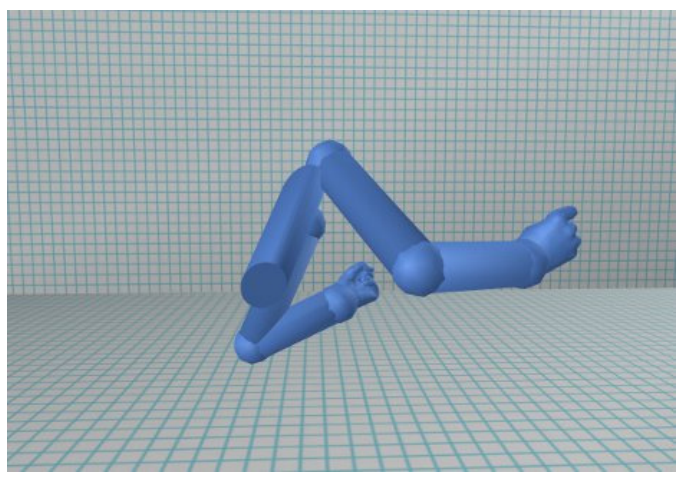

Figure 1. Prototype visualisation of a patient performing a reaching movement

The visualisation shows that the patient is compensating for difficulties in moving their arm by both raising their shoulder and moving their trunk forward. The simple stick figure representation allows both the therapist and the patient to look objectively at the movement (in comparison to the potential for an emotional response to viewing a video). The tool will allow the therapist to highlight the different compensatory movements and explain how they are reducing the effectiveness of the goals of the task.

In this scenario, the therapist then configures the tool to highlight those specific compensation strategies relevant for the patient. The patient is then asked to repeat the task several times with the visualisation tool displaying real-time visual indicators of when the patient is compensating during their movement.

A key change in this scenario for the interaction between the therapist and patient is that the tool performs the role of detecting and notifying the patient of their compensation strategies. The therapist may choose to augment the notifications with verbal encouragement, but is free to observe other aspects of the movement and to engage with the patient in other ways not related to the immediate task.

\section{B. Scenario 2: Communication and discussion of the patient's progress towards their rehabilitation goals}

In this scenario, the tool is being used for the diagnosis and tuning of an Ankle Foot Orthosis (AFO) following stroke. The patient and therapist will use the visualisations across four sessions: at baseline for diagnosis; during the tuning session; and at follow-up consultations at 3 months and 6 months.

In the patient's first session with the therapist, the patient will be asked to perform some simple walking movements in order to capture baseline measurements of their capability. This 
will be used as a benchmark for tracking and communicating the patient's progress through their rehabilitation. The same measurements will be repeated periodically during the rehabilitation process.

The visualisation tool will enable the data captured at the different sessions to be directly compared to assess where any improvements in capability have been achieved. Progress through rehabilitation can be complex - there may be periods of rapid improvement, but also times where improvement is small, or there may be intermediate reductions in capability. The use of the visualisation tool will be investigated in assisting the therapist to manage the expectations of the patient by showing in detail what could be achieved through rehabilitation, the probability of different outcomes and set out a personalised plan of intermediate rehabilitation goals. The following screenshots demonstrate an early prototype version of the tool to support this discussion.

In Figures 2 and 3, the patient's baseline measurements have been recorded in their first session with the therapist. The interrelated factors contributing to their slow and uncomfortable gait pattern can be shown visually and discussed between the patient and the therapist. The tool enables the therapist to dynamically highlight different aspects of the gait pattern which are most important to address for the patient.

In Figure 2, the therapist has selected the visualisation to show the unevenness of the gait and the small step length which is contributing to slow walking speed.

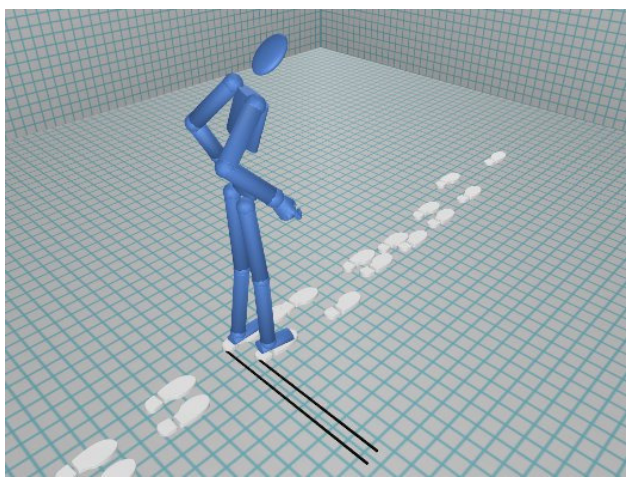

Figure 2. Visualisation of baseline gait measurement before the fitting of an Ankle Foot Orthosis. This viewpoint highlights the unevenness of the gait and the small step length which is contributing to slow walking speed

In Figure 3, the therapist has switched the viewpoint to a close up of the knee to show where the knee is going into hyperextension i.e. the knee joint is going outside its normal range of motion.

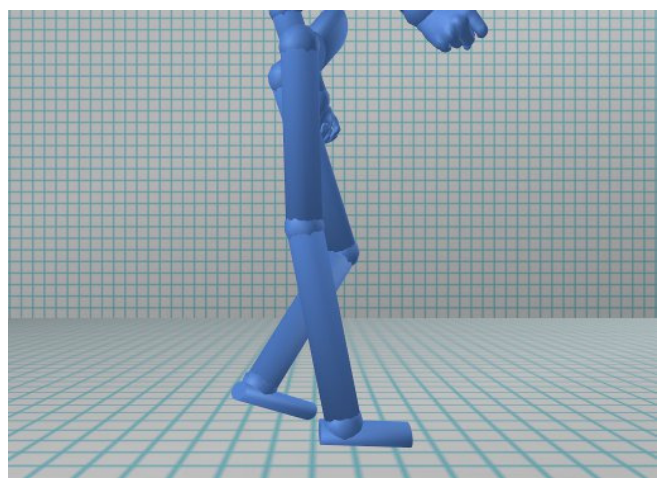

Figure 3. Visualisation of the patient's hyperextension of the right knee joint during walking motion before the fitting of an Ankle Foot Orthosis

Figure 4 provides a sample visualisation of the improvement in gait pattern a patient has achieved from using an AFO between their baseline measurement and their six month follow-up.

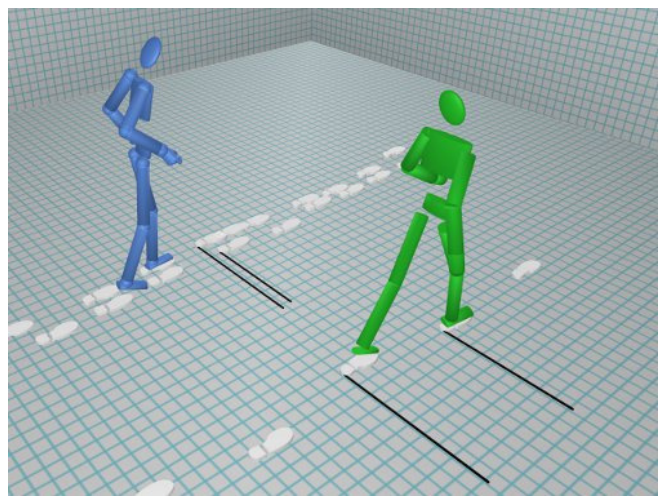

Figure 4. Visualisation of uneven and slow gait of stroke survivor at baseline (blue figure) relative to improved gait symmetry and walking speed at 3 month follow-up (green figure).

Using the visualisation tool in this scenario, the patient and therapist can both see in detail how the patient's gait has improved between sessions. The potential of displaying the progress objectively in this way may elicit clearer discussion of any different opinions between the patient and the therapist on the progress being made in the rehabilitation [8]. For instance, the patient may feel positive about the improvement, but the therapist may consider that the walking pattern is still not correct and may cause long term problems. The opposite may be true and the patient may be disappointed by the progress, yet the therapist may think that good progress has been made for the time period. The hypothesis is that the immediacy of the visualisation method, and the ability to clearly highlight different components of the movement and how they relate to each other will enable the mediation of such concerns and inform the next steps of the rehabilitation process.

\section{Scenario 3: Independent use of the visualisation tool by the patient in the home to maintain motivation and adherence to their rehabilitation tasks}

In this scenario, the patient has recently had Total Knee Replacement surgery and has been prescribed a number of 
home-based exercises to perform regularly during the day in order to regain strength and range of motion at the joint. The patient is supplied with wearable motion sensors (under development by our partners at Glasgow Caledonian University) and a laptop running visualisation software which they can use at their own convenience.

There are a number of challenges in providing technology for the home environment - particularly the user acceptance of the technology and the use of the equipment by the patient without the therapist to set up and troubleshoot problems, as found in [7]. These issues are essential to consider but will not be discussed in this paper in order to focus on the implications of using the visualisation tool in the home environment for the interaction between the patient and the therapist.

As the exercises only involve the working of the knee joint in isolation, the participant can use only two sensors rather than capture the motion of the whole body i.e. one attached to the upper leg, one attached to the lower leg. The tool will enable the patient to get immediate feedback on their performance during the exercises and to self-manage their own progress. Two early prototypes of how the patient's exercise performance could be represented are shown in Figure 5.

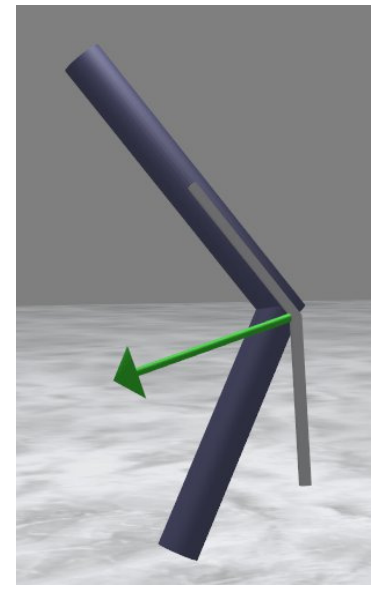

(a)

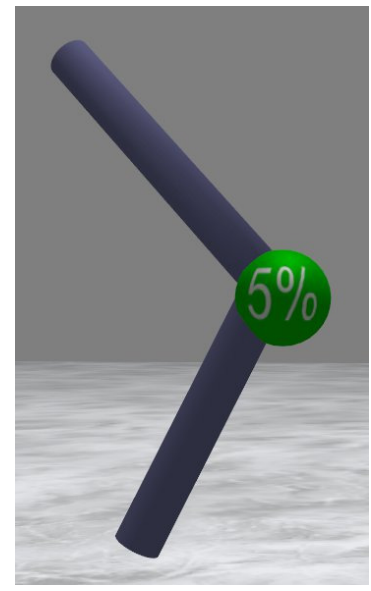

(b)
Figure 5. Two examples of the real-time visualisation of an exercise to improve range of motion at the knee. (a) The green arrow overlay on the limb segments represents the target knee angle the participant has to try and reach. The grey line indicates the maximum angle the participant could reach 4 weeks ago. (b) The percentage improvement in knee angle from the previous session is overlaid on the limb segments as the participant performs the exercise.

The primary advantage which the availability of rehabilitation tools in the home may offer is that the tool can be accessed at any time. With the clinical models discussed in scenarios 1 and 2 the interaction of the patient with the tool will be more occasional, possibly months apart.

As a consequence, the therapist will not always be available during the exercises. An effective home-based tool will need to encapsulate some of the knowledge of the therapist within the tool to compensate for a reduction in contact with the therapist. An example of this would be the need to automatically adjust the goals of the rehabilitation tasks in response to patient performance and fatigue. The extent to which a rule-set can be determined for how the tool should automatically adjust its parameters in response to the patient will be task dependent.

The study will also explore the ability to establish a relationship remotely between the patient and the therapist, and whether the richness of the patient-therapist dialogue in a clinical or community setting can be partially retained.

To facilitate this, the patient will be able to send their recorded movements to their therapist for analysis. The therapist will be able to remotely adjust the configuration of the exercises for the patient, and arrange for further discussions on their rehabilitation plan remotely using webcam or audio communications.

\section{CONCLUSIONS}

The on-going development of the visualisation tool as described in this paper will be used to investigate the potential to enhance the dialogue between patients and therapists by improving the communication of the correct way to perform rehabilitation tasks, how they relate to the patient's individual rehabilitation goals and how their rehabilitation is progressing. During the trials, the dialogues between patients and therapists in the intervention and control groups will be captured using a mix of observation and interviews with the participants.

The use of new rehabilitation tools and technology suitable for the home environment may empower the patient to better manage and customize their own rehabilitation process. Further, there may be cost savings possible by reducing the time spent with therapists in expensive-to-operate clinical environments.

However the opportunities for the use of technology to enhance a patient's interactions with their therapist rather than replace it should also be explored.

\section{ACKNOWLEDGMENTS}

The authors would like to thank the greater envisage project team. This research is funded by the MRC Lifelong Health and Wellbeing programme (LLHW), grant number G0900583. LLHW is a cross council initiative in partnership with the UK health departments and led by the MRC.

\section{REFERENCES}

[1] C. Teutsch, "Patient-doctor communication," Medical Clinics of North America, vol. 87, 2003, p. 1115-1146.

[2] B. Durward, G. Baer, and P. Rowe, "Functional Human Movement: Measurement and Analysis," 1997.

[3] A.S. Macdonald, D. Loudon, C. Docherty, and E. Miller, Project findings: Innovation in envisioning dynamic biomechanical data to inform healthcare and design guidelines and strategy, New Dynamics of Ageing Programme, Sheffield: 2009. 
[4] P. Craig, P. Dieppe, S. Macintyre, and S. Michie, "Developing and evaluating complex interventions: the new Medical Research Council guidance," British Medical Journal, 2008.

[5] S. Lewin, C. Glenton, and A.D. Oxman, "Use of qualitative methods alongside randomised controlled trials of complex healthcare interventions: methodological study," Bmj, vol. 339, Sep. 2009, p. b3496-b3496.

[6] A S. Macdonald, D. Loudon, P.J. Rowe, D. Samuel, V. Hood, a C. Nicol, M. a Grealy, and B. a Conway, "Towards a design tool for visualizing the functional demand placed on older adults by everyday living tasks," Universal Access in the Information Society, vol. 6, Aug. 2007, pp. 137-144.

[7] G. Mountain, S. Wilson, C. Eccleston, S. Mawson, J. Hammerton, T. Ware, H. Zheng, R. Davies, N. Black, N. Harris, T. Stone, and H. Hu, "Developing and testing a telerehabilitation system for people following stroke: issues of usability," Journal of Engineering Design, vol. 21, Apr. 2010, pp. 223-236.

[8] R. Wiles, A. Ashburn, S. Payne, and C. Murphy, "Patients' expectations of recovery following stroke: a qualitative study.," Disability and rehabilitation, vol. 24, Nov. 2002, pp. 841-50.

[9] R. Adams, "Improving health outcomes with better patient understanding and education," Risk Management and Healthcare Policy, Oct. 2010, p. 61 .
[10] R.C. Van De Weyer, C. Ballinger, and E.D. Playford, "Goal setting in neurological rehabilitation: staff perspectives.," Disability and rehabilitation, vol. 32, Jan. 2010, pp. 1419-27.

[11] R.C. Holliday, C. Ballinger, and E.D. Playford, "Goal setting in neurological rehabilitation: patients' perspectives.," Disability and rehabilitation, vol. 29, Mar. 2007, pp. 389-94.

[12] P. Isenberg, T. Zuk, C. Collins, and S. Carpendale, "Grounded evaluation of information visualizations," Proceedings of the 2008 conference on BEyond time and errors novel evaLuation methods for Information Visualization - BELIV '08, 2008, p. 1.

[13] R. Mazza and A. Berrè, "Focus group methodology for evaluating information visualization techniques and tools," Proceedings of the 11th IEEE International Conference on Information Visualisation, Zurich 2-7 July: 2007, pp. 74-80.

[14] S. Faisal, P. Cairns, and A. Blandford, "Challenges of evaluating the information visualisation experience," Proceedings of the 21st British HCI, British Computer Society, 2007.

[15] Distance Lab. "Remote Rehabilitation." <http://www.distancelab.org/projects/remote-rehabilitation/index.html> 